\title{
3D Atomic Scale Quantification of Nanostructures and their Dynamics Using Model-based STEM
}

Sandra Van Aert ${ }^{1}$, Annick De Backer ${ }^{1}$, Annelies De wael ${ }^{1}$, Jarmo Fatermans ${ }^{1}$, Thomas Friedrich ${ }^{1}$, Ivan Lobato $^{1}$, Colum O’Leary ${ }^{2}$, Aakash Varambhia ${ }^{3}$, Thomas Altantzis ${ }^{1}$, Lewys Jones ${ }^{4}$, Arjan den Dekker ${ }^{5}$, Peter Nellist ${ }^{2}$ and Sara Bals ${ }^{1}$

${ }^{1}$ EMAT, NANOLab Center of Excellence, University of Antwerp, Antwerp, Antwerpen, Belgium, ${ }^{2}$ University of Oxford, Oxford, England, United Kingdom, ${ }^{3}$ Department of Materials, University of Oxford, Oxford, England, United Kingdom, ${ }^{4}$ Trinity College Dublin, Dublin, Dublin, Ireland, ${ }^{5}$ imecVision Lab, University of Antwerp, Wilrijk, Antwerpen, Belgium

Quantitative 3D characterization at high resolution is often required in order to fully exploit structureproperty relations of nanomaterials. In recent years, the resolution of electron tomography has reached the atomic scale. However, for a successful 3D reconstruction using electron tomography multiple exposures are required, which is not always feasible when studying, for example, radiation-sensitive nanostructures. In order to overcome these limitations, an alternative method can be used where the 3D atomic structure is reconstructed from atom counts obtained from a single ADF STEM projection image. These atom counts can be used to create an initial atomic model which serves as an input for an energy minimization to obtain a relaxed $3 \mathrm{D}$ reconstruction of a nanostructure [1].

To count atoms with single-atom sensitivity, a minimally required electron dose is necessary, while on the other hand the risk of knock-on damage, induced by the high energy electrons, puts an upper limit on the tolerable dose. An important challenge is therefore to develop experimental strategies to optimize the electron dose by balancing atom-counting fidelity versus the risk of knock-on damage. To achieve this goal, a statistical framework combined with physics-based modelling of the dose-dependent processes is proposed and experimentally verified. This model enables an investigator to theoretically predict, in advance of an experimental measurement, the electron dose resulting in an unambiguous quantification of nanostructures in their native state with the highest attainable precision [2].

For beam-sensitive materials, where the optimal electron dose can be low, the images will exhibit a limited signal-to-noise ratio and additionally, they will show a very weak contrast in the presence of light elements. In order to reliably detect the presence or absence of atomic columns and even single atoms, the maximum a posteriori (MAP) probability rule is proposed as a model-order selection method in which a Bayesian framework is used [3]. To illustrate this, figure 1(a) shows part of a synthetic ADF STEM image of graphene, artificially created from an experimental 4D STEM dataset. By using MAP selection, the probabilities for increasing number of carbon atoms present in this image can be calculated. Figure 1(b) shows this probability curve, where the obtained probabilities are compared to each other in a relative manner. The curve reaches its maximum at 64 indicating that the most probable atomic structure consists of 64 carbon atoms. The refined parametric model is shown in figure 1(c), clearly revealing the hexagonal lattice of graphene without imposing prior knowledge concerning the expected atomic structure. In addition, in recent work we investigated the use and potential benefit of deep convolutional neural networks to restore low dose, scan noise distorted ADF STEM images.

Our method of atom-counting opens up new opportunities to measure variations of the 3D atomic structure of nanoparticles under the flow of a selected gas [4]. In situ experiments clearly demonstrate that many 
questions in materials science require a quantification of the material's dynamics from a series of sequentially recorded images. However, the standard approach for atom-counting, where each frame is analyzed individually, does not take advantage of the time aspect available in a series of ADF STEM images. We therefore extended the atom-counting method using the so-called factorial hidden Markov model. This new method is very promising for revealing atomic scale dynamics [5]. For example, when analyzing a series of ADF STEM projection images of a Pt nanoparticle, shown in figure 2(a), we can reliably follow the evolution of the number of atoms as a function of time, see figure 2(b). While the total number of $\mathrm{Pt}$ atoms remains the same, the obtained atom counts clearly indicate that because of the presence of beam-induced surface diffusion, the nanoparticle gradually loses its faceted morphology during irradiation. Moreover, this hidden Markov model analysis has the added advantage that it enables us to quantify the probability for surface diffusion. In conclusion, new developments in the field of quantitative STEM will be presented enabling one to reliably quantify dynamic structural changes resulting from adatom dynamics, surface diffusion, beam effects or during in situ experiments [6].

(a)

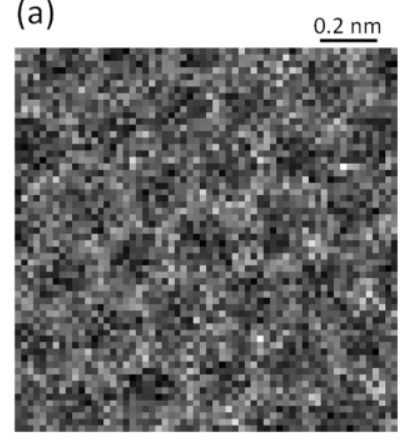

(b)

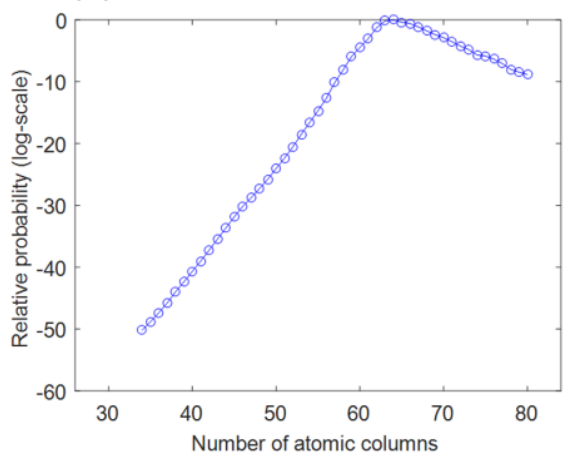

(c)

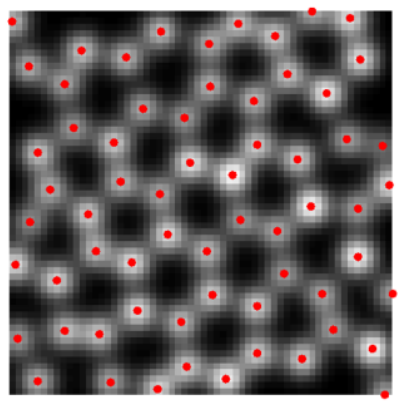

Figure 1. (a) Synthetic ADF STEM image of graphene obtained from an experimental 4D STEM dataset. (b) MAP probability rule evaluated for the experimental data shown in (a). (c) Most probable parametric model of the experimental data in (a) following the MAP probability rule in (b).

(a)

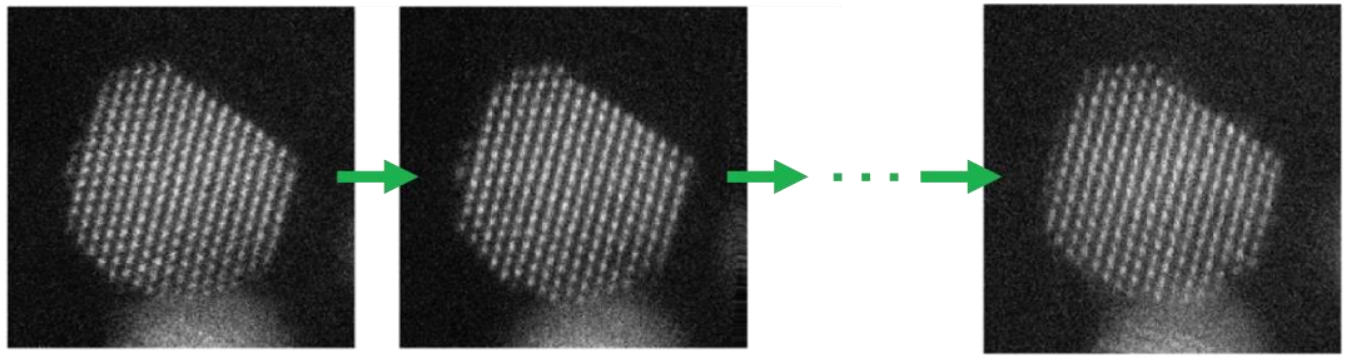

(b)

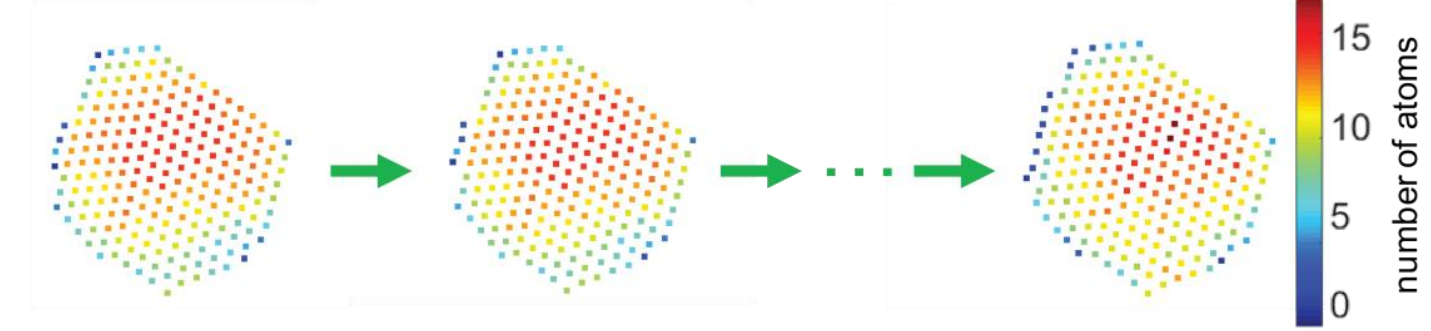

Figure 2. (a) Experimental ADF STEM time series of a Pt nanoparticle. (b) Estimated atom counts using a hidden Markov model. 


\section{References}

[1] A De Backer et al., Nanoscale 9 (2017), p. 8791.

[2] S. Van Aert et al., Physical Review Letters 122 (2019), 066101.

[3] J. Fatermans et al., Physical Review Letters 121 (2018), 056101.

[4] T. Altantzis et al., Nano Letters 19 (2019), p. 477.

[5] A. De wael et al., Physical Review Letters (2020), accepted for publication.

[6] This work was supported by the European Research Council (Grant 770887 PICOMETRICS to SVA and Grant 815128 REALNANO to SB, Grant 823717 ESTEEM3). The authors acknowledge financial support from the Research Foundation Flanders (FWO, Belgium) through project fundings, a postdoctoral grant to $\mathrm{ADB}$ and a doctoral grant to $\mathrm{ADw}$. 